\title{
How to Write the Discussion?
}

That then is the first draft and you should never think of having fewer than six drafts

Stephen Lock, BMJ editor in chief (1929-...)

\subsection{What Is the Importance of the Discussion?}

Many authors, and editors, think this is the most difficult part of a paper to write well and have described it variously to be the 'narrating the story of your research', 'the movie or the main scientific script' and the 'proof of the pudding'. The idea of a discussion is to communicate to the readers the importance of your observations and the results of all your hard work. In this section, you are expected to infer their meaning and explain the importance of your results and finally provide specific suggestions for future research $[1,2]$. The discussion places the outcome into a larger context and mentions the implications of the inferences for theoretical and practical purposes [3]. 


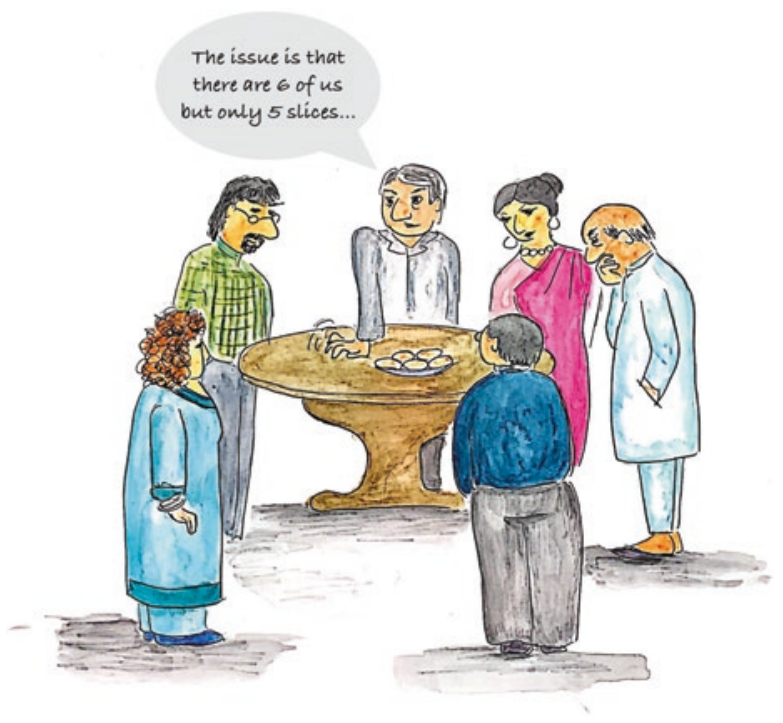

\subsection{How Should I Structure the Discussion Section?}

There are three major portions for the discussion of a manuscript.

The first paragraph should baldly state the key findings of your research. Use the same key concept you gave in the introduction. It is generally not necessary to repeat the citations which have already been used in the Introduction. According to the 'serial position effect', themes mentioned at the beginning and end of a paragraph are more likely to be remembered than those in the middle [1]. However, one should remember that the discussion should not look like a second introduction, and all the ancillary information which has been previously cited should not be repeated [4].

For example, in a paper on the 'Role of sulfasalazine in the Chikungunya arthritis outbreak of 2016', the review may start with, 'Our key findings suggest that chikungunya arthralgia is a self-limiting disorder. Persistent arthritis was recorded in only $10 \%$ of the affected population and in those who received sulfasalazine, clinical improvement both in tender and swollen joints, was recorded in 95\% of the subjects'.

The middle portion should consist of the body of the discussion. This section interprets the important results, discusses their implications and explains how your data is similar to or different from those that have been published previously.

Discuss in fair detail studies supporting your findings and group them together, against those offering a different perspective (e.g., Western experience, smaller numbers, non-randomized studies, etc.). An explanation should be offered on how your work is similar to others or how it is different from the others. This should be followed by a review of the core research papers. The results should now be divided thematically and analyzed. The discussion should also contain why the study is new, why it is true, and why it is important for future clinical practice [4-6]. 
For the above research mention the clinical features, patterns of joint involvement, how long arthritis persisted, and any role of disease-modifying agents. Have any other researchers found different findings under the same circumstances.

The final paragraph should include a 'take home message' (about one or two) and point to future directions for investigation that have resulted from this study.

The discussion can be concluded in two ways:

- By again mentioning the response to the research question $[5,7]$

- By indicating the significance of the study $[2,4]$

You can use both methods to end this section. Most importantly you should remember that the last paragraph of the discussion should be 'strong, clear, and crisp' and focus on the main research question addressed in the manuscript. This should be strengthened by the data which clearly states whether or not your findings support your initial hypothesis [1, 5, 8-10].

\subsection{What Are the General Considerations for Writing a Discussion? $[3,10,11]$}

- Start the discussion with the 'specific' problems and move to the 'general' implications (Fig. 21.1).

- The discussion should not look like a mass of unrelated information. Rather, it should be easy to understand and compare data from different studies.

- Include only recent publications on the topic, preferably from the last 10 years.

- Make certain that all the sources of information are cited and correctly referenced.

- Check to make sure that you have not plagiarized by using words quoted directly from a source.

Fig. 21.1 How a

discussion should look.

First arrow-Mention your

key results/findings;

Second arrow-Discuss

your results with their

explanations/step by step;

Third Arrow-Enumerate

your studies limitations

and strengths; Last

arrow-Suggest future

directions for investigation

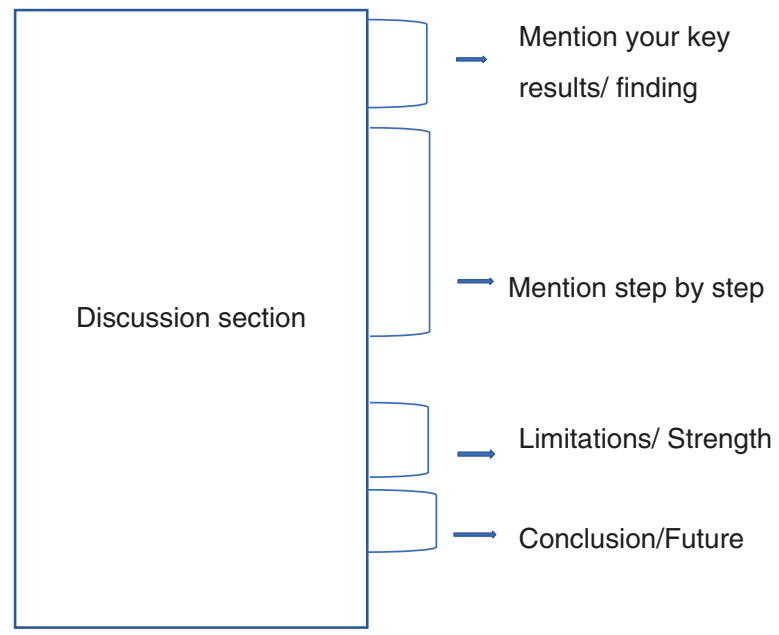


- The written text written should be easily understood, crisp, and brief. Long descriptive and informal language should be avoided.

- The sentences should flow smoothly and logically.

- You need not refer to all the available literature in the field, discuss only the most relevant papers.

\subsection{Discussion Is Not a War of Words}

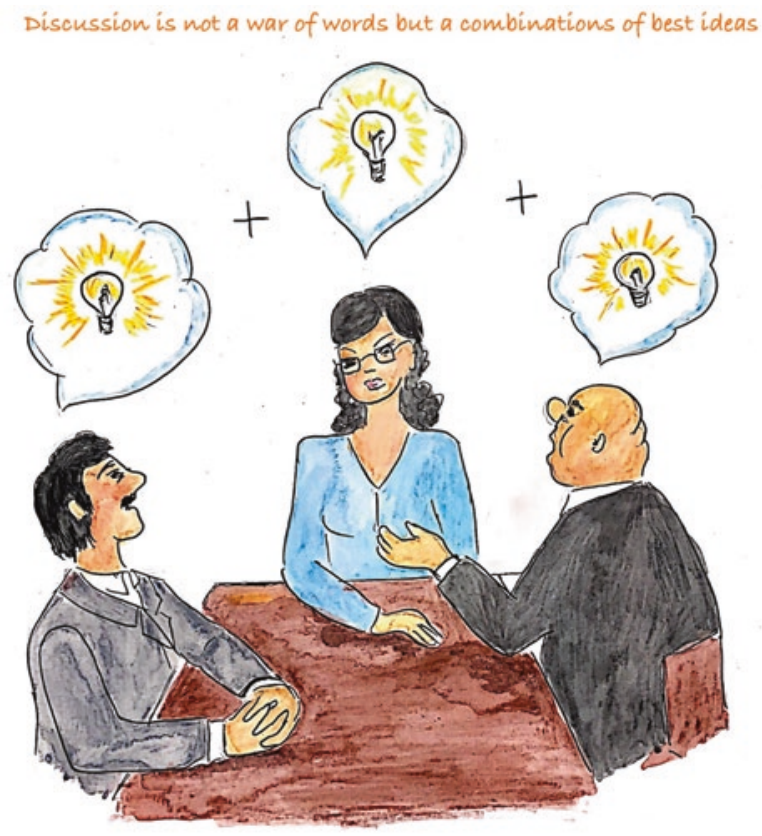

\subsection{How Long Should the Discussion in the Manuscript Be?}

Most journals do not mention any limits for discussion as long as it is brief and relevant (Fig. 21.2). As a rule, 'The length of the discussion section should not exceed the sum of other parts-introduction, materials and methods, and results'. [3] In any good article, the discussion section is 3-4 pages, 6-7 paragraphs, or approximately 10 paragraphs, and $1000-1500$ words $[1,5,8,12]$.

\subsection{What Should Be Written in the Conclusion Section?}

The conclusion is the last paragraph and has the carry-home message for the reader. It is the powerful and meaningful end piece of the script. It states what change has the paper made to science and it also contains recommendations for future studies. 
Fig. 21.2 Discussion pyramid

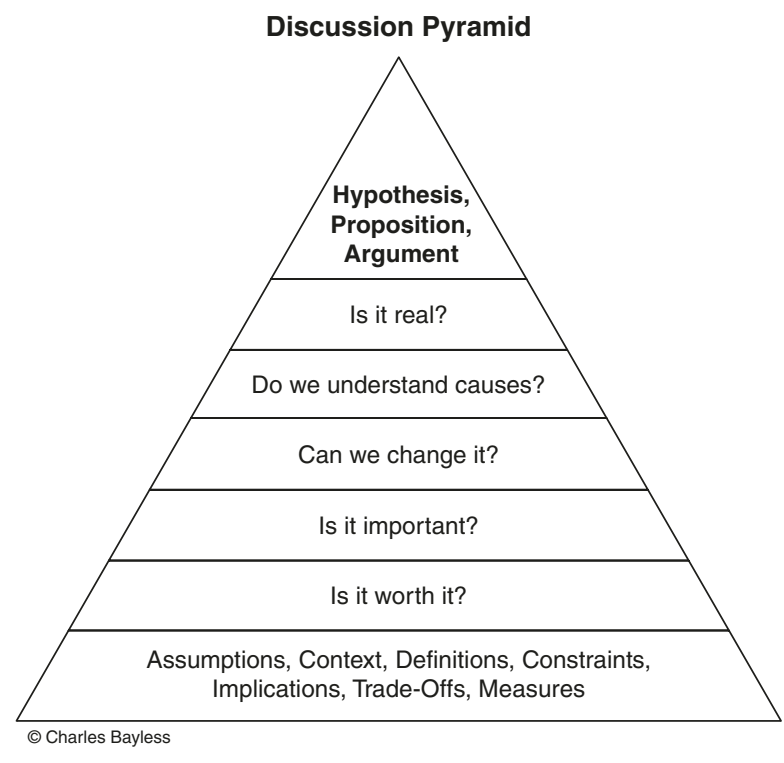

\subsection{Conclusions}

- Discussion is not a stand-alone section, it intertwines the objectives of the study with how and what was achieved.

- The major results are described and compared with other studies.

- The author's own work is critically analysed in comparison with that of others.

- The limitations and strengths of the study are highlighted.

\section{References}

1. Masic I. How to write an efficient discussion? Mediev Archaeol. 2018;72(4):306-7.

2. Bagga A. Discussion: the heart of the paper. Indian Pediatr. 2016;53(10):901-4.

3. Ghasemi A, Bahadoran Z, Mirmiran P, Hosseinpanah F, Shiva N, Zadeh-Vakili A. The principles of biomedical scientific writing: discussion. Int J Endocrinol Metab. 2019;17:e95415.

4. Zeiger M. Essentials of writing biomedical research papers. Canadian J Stud Discourse Writing. 2000;11:33-6.

5. Bavdekar SB. Writing the discussion section: describing the significance of the study findings. J Assoc Physicians India. 2015;63:40-2.

6. Foote M. The proof of the pudding: how to report results and write a good discussion. Chest. 2009;135(3):866-8.

7. Alexandrov AV. How to write a research paper? Cerebrovasc Dis. 2004;18(2):135-8.

8. Annesley TM. The discussion section: your closing argument. Clin Chem. 2010;56(11):1671-4.

9. Ng KH, Peh WC. Writing the discussion. Singap Med J. 2009;50:458-60.

10. Coverdale JH, Roberts LW, Balon R, Beresin EV. Writing for academia: Getting your research into print: AMEE guide No. 74. Med Teach. 2013;35:e926-34.

11. Araujo CG. Detailing the writing of scientific manuscripts: 25-30 paragraphs. Arq Bras Cardiol. 2014;102(2):e21-3.

12. Kearney MH. The discussion section tells us where we are. Res Nurs Health. 2017;40(4):289-91. 
Open Access This chapter is licensed under the terms of the Creative Commons Attribution 4.0 International License (http://creativecommons.org/licenses/by/4.0/), which permits use, sharing, adaptation, distribution and reproduction in any medium or format, as long as you give appropriate credit to the original author(s) and the source, provide a link to the Creative Commons license and indicate if changes were made.

The images or other third party material in this chapter are included in the chapter's Creative Commons license, unless indicated otherwise in a credit line to the material. If material is not included in the chapter's Creative Commons license and your intended use is not permitted by statutory regulation or exceeds the permitted use, you will need to obtain permission directly from the copyright holder. 